ARTICLE

https://doi.org/10.1038/s41467-018-07768-9

\title{
Muc5b overexpression causes mucociliary dysfunction and enhances lung fibrosis in mice
}

\author{
Laura A. Hancock ${ }^{1}$, Corinne E. Hennessy ${ }^{1}$, George M. Solomon², Evgenia Dobrinskikh ${ }^{1}$, Alani Estrella ,
} Naoko Hara1, David B. Hill (10 3,4, William J. Kissner ${ }^{3}$, Matthew R. Markovetz (10 ${ }^{3}$, Diane E. Grove Villalon ${ }^{5}$, Matthew E. Voss ${ }^{5}$, Guillermo J. Tearney ${ }^{6}$, Kate S. CarrolI ${ }^{7}$ Y Yunlong Shi ${ }^{7}$, Marvin I. Schwarz' ${ }^{1}$ William R. Thelin ${ }^{5}$, Steven M. Rowe ${ }^{2}$, Ivana V. Yang ${ }^{1}$, Christopher M. Evans ${ }^{1,8}$ \& David A. Schwartz (D) 1,8

The gain-of-function MUC5B promoter variant rs35705950 is the dominant risk factor for developing idiopathic pulmonary fibrosis (IPF). Here we show in humans that MUC5B, a mucin thought to be restricted to conducting airways, is co-expressed with surfactant protein C (SFTPC) in type 2 alveolar epithelia and in epithelial cells lining honeycomb cysts, indicating that cell types involved in lung fibrosis in distal airspace express MUC5B. In mice, we demonstrate that Muc5b concentration in bronchoalveolar epithelia is related to impaired mucociliary clearance (MCC) and to the extent and persistence of bleomycin-induced lung fibrosis. We also establish the ability of the mucolytic agent P-2119 to restore MCC and to suppress bleomycin-induced lung fibrosis in the setting of Muc5b overexpression. Our findings suggest that mucociliary dysfunction might play a causative role in bleomycininduced pulmonary fibrosis in mice overexpressing Muc5b, and that MUC5B in distal airspaces is a potential therapeutic target in humans with IPF.

\footnotetext{
${ }^{1}$ Department of Medicine, University of Colorado Denver, School of Medicine, Aurora, CO 80045, USA. ${ }^{2}$ Department of Medicine, University of Alabama at Birmingham, School of Medicine, Birmingham, AL 35294, USA. ${ }^{3}$ Marsico Lung Institute, University of North Carolina, Chapel Hill, NC 27599, USA. ${ }^{4}$ Physics and Astronomy, University of North Carolina, Chapel Hill, NC 27599, USA. ${ }^{5}$ Parion Sciences, Inc, Durham, NC 27713, USA. ${ }^{6}$ Wellman Center for Photomedicine and Department of Pathology, Massachusetts General Hospital, Boston, Massachusetts; Department of Pathology, Harvard Medical School, Massachusetts General Hospital, Boston, Massachusetts, USA. ${ }^{7}$ Department of Chemistry, The Scripps Research Institute, Jupiter, FL 33458, USA.

${ }^{8}$ Department of Immunology, University of Colorado Denver, School of Medicine, Aurora, CO 80045, USA. These authors contributed equally: Laura A. Hancock, Corinne E. Hennessy. Correspondence and requests for materials should be addressed to D.A.S. (email: david.schwartz@ucdenver.edu)
} 
diopathic pulmonary fibrosis (IPF) is a progressive fibrotic lung disease with a median survival of $3-5$ years $^{1}$ that worsens despite treatment ${ }^{2,3}$. A common gain-of-function $M U C 5 B$ promoter variant rs35705950 is the dominant risk factor, either genetic or environmental, for the development of $\mathrm{IPF}^{4,5}$. MUC5B is a major gel forming mucin in the lung that plays a key role in mucociliary clearance (MCC) and host defense ${ }^{5}$ that is secreted from proximal submucosal glands and distal airway secretory cells $^{6-8}$. The MUC5B promoter variant is associated with enhanced expression of the MUC5B transcript in lung tissue from unaffected subjects and patients with IPF $^{4,9}$. In patients with IPF, excess MUC5B protein is especially observed in epithelial cells in the respiratory bronchiole and honeycomb cyst ${ }^{7,8}$, regions of lung involved in lung fibrosis. However, it remains unclear how MUC5B leads to the development of IPF.

Here we show that MUC5B is produced in cells lining distal airways and honeycomb cysts in human IPF. We also show that Muc5b overproduction in the distal lungs of mice are associated with MCC dysfunction and exaggerates the development of fibrosis, and that this can be prevented by treatment with a mucolytic agent. Our findings show a causative, dose-dependent role for Muc5b in murine lung fibrosis, and thus support development of mucolytic intervention strategies for human disease.

\section{Results}

MUC5B/Muc5b in the distal lung and effects on fibrosis. In human IPF lung tissue, we found that MUC5B is co-expressed with surfactant protein $C$ in columnar epithelial cells lining honeycomb cysts (Fig. 1a) and in type 2 alveolar epithelia (Fig. 1b), indicating that cell types involved in lung fibrosis in the distal airspace also express MUC5B. Accordingly, to understand the effect of increased $M u c 5 b$ expression, such as that associated with the MUC5B promoter variant, we generated two lines of $\mathrm{C} 57 \mathrm{BL} / 6$ mice that overexpress full-length murine $M u c 5 b$ genomic transgenes. $\operatorname{Tg}(\mathrm{Scgb} 1 \mathrm{a} 1-\mathrm{Muc} 5 \mathrm{~b})$ overproduce Muc5b under the control of a mouse secretaglobin lal promoter fragment ${ }^{10}$, and $\operatorname{Tg}($ SFTPC-Muc5b) mice overproduce Muc5b under the control of a human surfactant protein $\mathrm{C}$ promoter fragment (Supplementary Fig. 1a,b). These non-targeted transgenic lines are referred to as Scgbla1-Muc5b $\mathrm{b}^{\mathrm{Tg}}$ and SFTPC-Muc5b ${ }^{\mathrm{Tg}}$, and constitutively overexpress Muc5b in specific lung locations in addition to endogenous Muc5b (Supplementary Fig. 1c- $\mathrm{f}$ ). Notably, Scgb1a1-Muc5b ${ }^{T g}$ mice produce Muc5b throughout the conducting airways, whereas SFTPC-Muc $5 \mathrm{~b}^{\mathrm{Tg}}$ mice produce Muc5b in the distal airways and alveoli (Fig. 1c and Supplementary Fig. 1c). Muc5b gene-deficient mice on a congenic C57BL/6J background ( $M u c 5 b^{-1-}$ ) were included to assess the effect of the absence of Muc5 $b^{10}$.

Collectively, these mouse lines allowed for robust gain-offunction and loss-of-function analyses of the effects of Muc5b production, and its effect in mouse models of pulmonary fibrosis.

Initially, mice were challenged with three intratracheal (IT) doses of bleomycin $(2.5 \mathrm{U} / \mathrm{kg}, 1.5 \mathrm{U} / \mathrm{kg}$, and $1.5 \mathrm{U} / \mathrm{kg})$ over 7 weeks to strongly simulate the temporal heterogeneity and progressive nature of human IPF $^{11}$. SFTPC-Muc5b $\mathrm{b}^{\mathrm{Tg}}$ mice express substantially higher concentrations of Muc5b than Scgbla1-Muc5b ${ }^{\mathrm{Tg}}$ mice (Supplementary Fig. 1d-f). Furthermore, following challenge with bleomycin, SFTPC-Muc5b $\mathrm{b}^{\mathrm{Tg}}$ mice experience significantly reduced survival than Scgbla1Muc5b ${ }^{\mathrm{Tg}}, \mathrm{Muc5} \mathrm{b}^{-1-}$, and wild-type control mice for each strain (Fig. 1d). To minimize survivor bias, we used a lower dose of bleomycin $(2.0 \mathrm{U} / \mathrm{kg}, 1.0 \mathrm{U} / \mathrm{kg}$, and $1.0 \mathrm{U} / \mathrm{kg})$ in SFTPC-Muc5b $\mathrm{b}^{\mathrm{Tg}}$ mice for subsequent studies.

Following bleomycin challenge, both Scgbla1-Muc5b ${ }^{T g}$ and SFTPC-Muc5 $\mathrm{b}^{\mathrm{Tg}}$ mice demonstrate substantial lung injury that was less prominent in $M u c 5 b^{-1-}$ mice (Fig. 1e). Therefore, we assessed lung fibrosis biochemically by measuring hydroxyproline
(HP) content, a marker of collagen deposition. Bleomycin challenged Scgbla1-Muc5 $b^{T g}$ and SFTPC-Muc5b ${ }^{\text {Tg }}$ mice have elevated amounts of lung HP compared to their transgene negative littermate controls (Fig. 1f). In contrast, mice lacking Muc5b $\left(\mathrm{Muc5}^{-1-}\right)$ demonstrate decreased HP compared to wild-type littermates (Fig. 1f). This difference in collagen was quantified using confocal/multiphoton-excitation fluorescence microscopy with second harmonic generation (SHG, Fig. 1g). Quantification of SHG images demonstrates that Scgbla1Muc5b $5 b^{T g}$ and SFTPC-Muc5b $b^{\text {Tg }}$ mice have significantly more collagen than non-challenged and transgene-negative littermate controls following challenge with bleomycin (Fig. 1h, i). Thus, $M u c 5 b$ expression correlates with fibrosis detected biochemically and histologically, suggesting that the presence of excess Muc5b enhances the fibrotic response to bleomycin and that absence of Muc5b diminishes this response.

Effects of Muc5b in distal airspaces on MCC. To explore a mechanism involved in the Muc5b-associated fibrotic response to bleomycin, we considered the effect of enhanced expression of Muc5b on MCC ${ }^{12,13}$. Examination of the tracheal mucus layer by micro optical coherence tomography ( $\mathrm{OOCT})^{14-16}$ shows distinct differences in mucociliary transport apparatus between SFTPCMuc5b $b^{T g}$ and transgene negative littermates (Fig. 2a and Supplementary videos 1 and 2). Quantitatively, the mucus depth is significantly greater (Fig. 2b) in SFTPC-Muc $5 b^{\text {Tg }}$ mice compared to transgene-negative littermate control mice, but this has a minimal effect on the periciliary layer depth (Fig. 2c), suggesting that osmotic forces transmitted by increased mucus concentration are not substantial ${ }^{12}$. However, both ciliary beat frequency (Fig. 2d) and mucociliary transport rate (Fig. 2e) are disrupted in SFTPC-Muc5b ${ }^{\mathrm{Tg}}$ mice. Mucin overexpression in Scgbla1Muc5b $b^{T g}$ mice induces similar $\mu$ OCT results (Supplementary Fig. 2, Supplementary videos 3 and 4), although both the mucus layer increase vs. respective littermate controls (7.0 in Scgbla1-

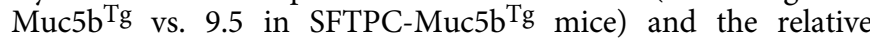
mucociliary transport decrement $(49.5 \%$ and $70.6 \%$, respectively) are less pronounced in this model, consistent with bleomycin sensitivity (Fig. 1d-i).

Effects of mucolytic treatment on MCC and fibrosis. The increase in fibrosis and decrease in mucociliary transport associated with elevated Muc5b levels led us to investigate whether therapeutic agents predicted to accelerate mucus clearance would be effective in improving outcomes in the SFTPC-Muc5b $\mathrm{b}^{\mathrm{Tg}}$ mouse model of lung fibrosis. Therefore, we tested the effects of a mucolytic compound, P-211917 (Parion Sciences, Durham, NC), that is designed to hydrolyze disulfide bonds more efficiently than existing reducing agents, e.g. N-acetylcysteine (NAC). P-2119 yields faster disulfide bond reduction and at lower concentrations than NAC in assays utilizing a model disulfide bond substrates (5,5'-dithiobis-2-nitrobenzoic acid (Fig. 3a, b) and MUC5B purified from human saliva (Fig. 3c). Additionally, in concentrated human airway mucus the ability of P-2119 to reduce MUC5B (Fig. 3d) causes improvements in viscosity and elasticity that are consistent with mucus alterations predicted to facilitate clearance (Fig. 3e, f and Supplementary Fig. 3) and are directly related to decreased MUC5B molecular mass determined by multi-angle laser light scattering spectroscopy (Fig. $3 \mathrm{~g}$ ). With these studies demonstrating the effectiveness of a mucolytic agent in vitro, we next sought to assess the effectiveness of P-2119 in vivo. In WT C57BL/6J mice challenged with LPS to induce Muc5b expression and acute lung injury, P-2119 inhalation effectively depolymerizes Muc5b, and the mucolytic effect of P-2119 persists for $120 \mathrm{~min}$ (Fig. 3h). Having established dose and time course parameters in WT mice, we next tested the effects of P-2119 in SFTPC-Muc5b ${ }^{\text {Tg }}$ mice treated with a single IT dose of bleomycin. In these animals, 
a

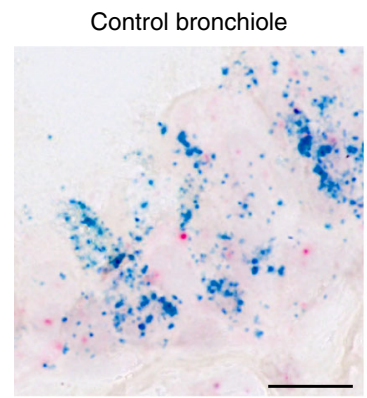

C

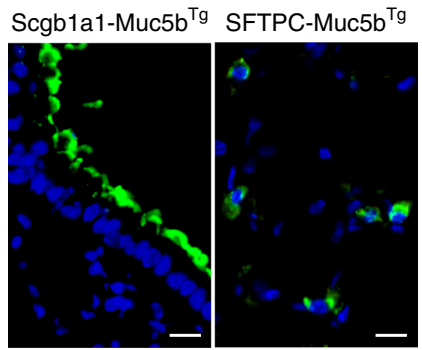

g
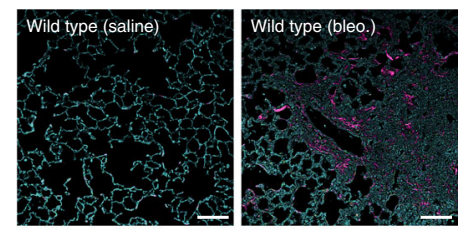

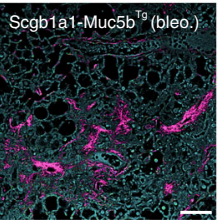

IPF bronchiolar cyst

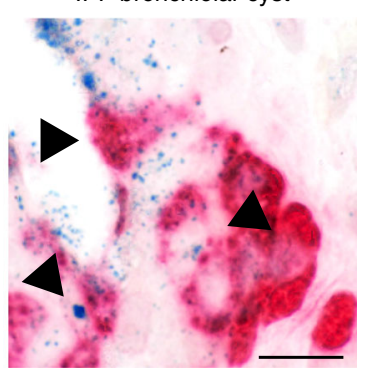

d

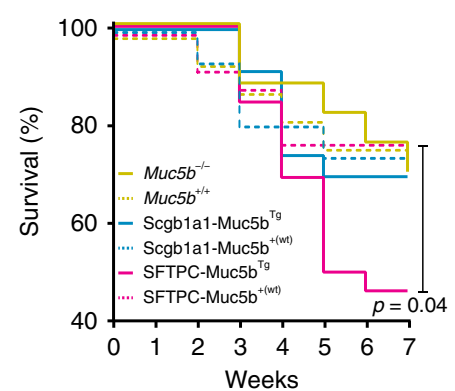

b

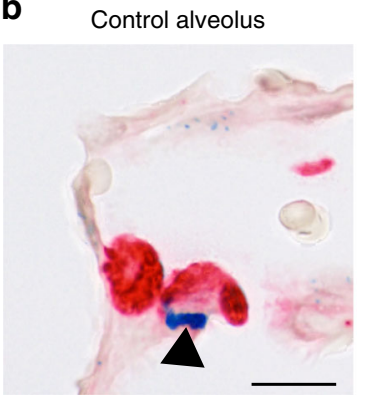

IPF alveolar cyst

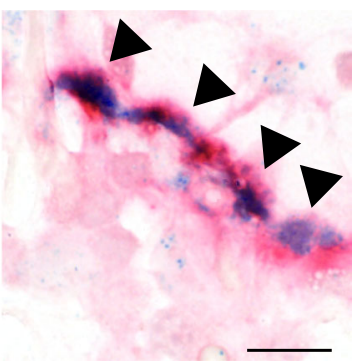

-

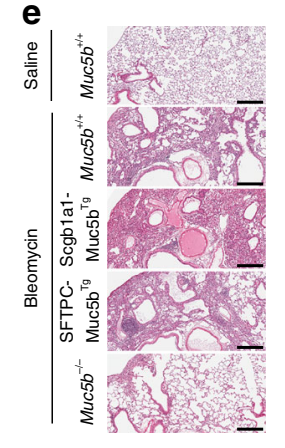

$\mathbf{f}$

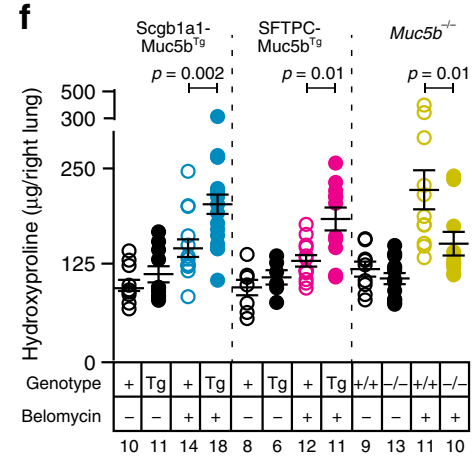

h

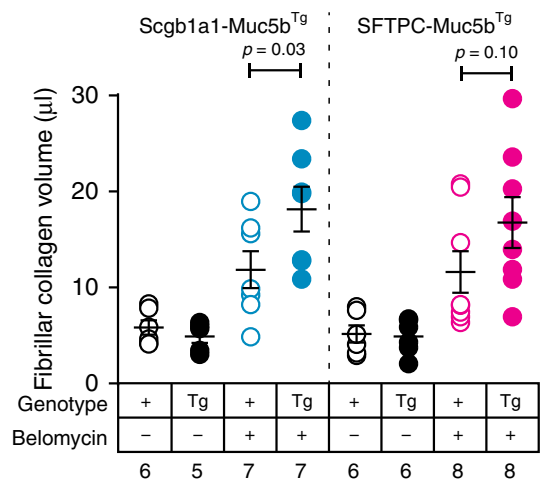

i

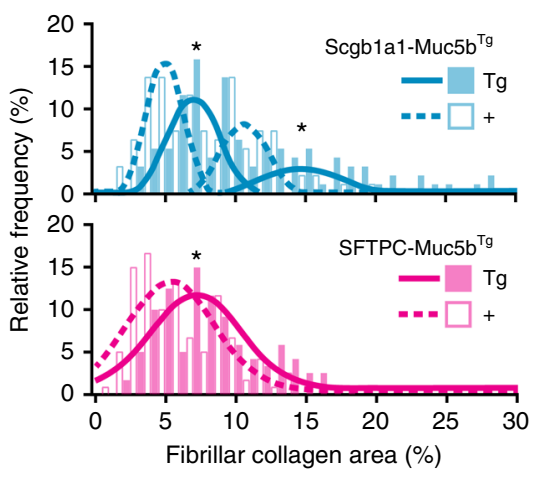

Fig. 1 Muc5b overexpression in the distal lung is associated with greater fibrogenesis following bleomycin. $\mathbf{a}$, $\mathbf{b}$ In situ hybridization of human lung specimens from control and IPF subjects with MUC5B variant rs35705950. Arrowheads depict cells co-expressing SFTPC (red) and MUC5B (blue) in control bronchioles and IPF bronchiolar structures a and in control type 2 alveolar epithelia and IPF type 2 alveolar structures $\mathbf{b}$. c Transgenic mice expressing Muc5b under control of the mouse Scgbla1 and the human SFTPC promoters demonstrate Muc5b protein in conducting airways and in type 2 alveolar epithelia. d, e After repeated doses of bleomycin $\left(2.5 \mathrm{U} / \mathrm{kg}\right.$, IT on day $0,1.5 \mathrm{U} / \mathrm{kg}$ on days 14 and 28), SFTPC-Muc5b $\mathrm{b}^{\mathrm{Tg}}$ demonstrated worse

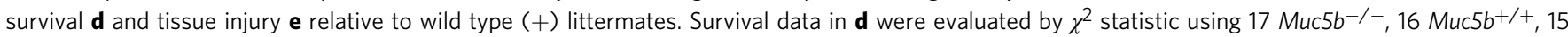
Scgb1a1- Muc5bTg, 23 Scgb1a1-Muc5b+(wt), 26 SFTPC-Muc5b $b^{T g}$, and 25 SFTPC-Muc5b+(wt), mice. f-i Scgb1a1- Muc5b $b^{T g}$, Muc5b-/-, and control mice were treated IT with bleomycin as in d. To induce similar levels of fibrosis while limiting survivor bias, SFTPC-Muc5b $5 \mathrm{~g}$ and controls received $2.0 \mathrm{U} / \mathrm{kg}$ bleomycin on day 0 , followed by $1.0 \mathrm{U} / \mathrm{kg}$ on days 14 and 28 . HP content increased in Scgbla1-Muc5b $\mathrm{b}^{\top \mathrm{g}}$ and SFTPC-Muc5b $\mathrm{b}^{\mathrm{Tg}}$ but decreased in Muc5b-/mice compared to wild type $(+)$ controls for each strain. $\mathbf{g}$-i Fibrillar collagen (magenta in $\mathbf{g}$ ) was assessed in peripheral lung tissues by confocal/multiphoton fluorescence microscopy with SHG. h, i Fibrillar collagen volumes following bleomycin were increased per mouse $\mathbf{h}$ and showed heterogeneous distributions $\mathbf{i}$ in Tg mice compared to controls. Data in $\mathbf{i}$ were analyzed by $t$-test $\left(n=105\right.$ Scgbla1-Muc5b $b^{\top g}$ and 105 Scgbla1-Muc5b $+(w t)$ images, and $n=$

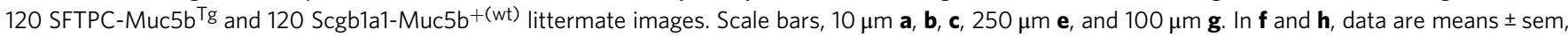
numbers in italics indicate $n$ animals used per experiment, and $p$-values indicate differences determined by ANOVA with Holm-Sidak's multiple comparisons test. In $\mathbf{i}{ }^{*}$ indicates statistical significance $\left(p<10^{-5}\right)$

P-2119 treatment also results in smaller mucin polymers detected in lung lavage fluid (Fig. 3i). Mucolytic treatment results in acute clearance of inflammatory cells from the lungs, which is demonstrated by a significant and rapid diminishment in lung lavage leukocyte numbers (Fig. $3 \mathrm{j}$ and Supplementary Fig. 4) concurrent with Muc5b depolymerization. In aggregate, these results suggest that P-2119, a mucolytic agent, may favorably influence mucus properties in the airspace by improving MCC, consequently minimizing fibrosis following bleomycin-induced lung injury in the context of Muc5b overproduction.

To pursue this further, we focused on the SFTPC-Muc $5 b^{T g}$ mice that produced the highest concentrations of Muc5b (see Supplementary Fig. 1d,f), were most responsive to bleomycin (Fig. 1), and had more pronounced changes in mucociliary transport (see Fig. 2 and Supplementary Fig. 2). SFTPC-Muc5b ${ }^{\text {Tg }}$ mice were challenged with $2.5 \mathrm{U} / \mathrm{kg}$ of bleomycin IT, were 
allowed to respond to bleomycin for 1 or 8 weeks, and were then exposed to 2 weeks of aerosolized P-2119. Hence, mice were observed at 3 or 10 week endpoints post-bleomycin (Fig. 4a). This comprehensive approach allowed us to test the ability of P-2119 to treat both short and long-term fibrotic lung responses induced by bleomycin, whereas leaving the effects of bleomycin on lung injury intact ${ }^{18}$. Treatment with P-2119 reduces the severity of chronic inflammation (Fig. 4b, Supplementary Fig. 5), as well as mortality associated with bleomycin-induced lung injury (Supplementary Fig. 6). These protective effects of P-2119 on lung injury are not associated with changes in redox balance (Supplementary Fig. 7) or inflammatory cytokine/chemokine expression (Supplementary Table 1).

To assess the anti-fibrotic effects of mucolytic treatment, collagen levels in mouse lung tissues were quantified using biochemical and histological assays. Whereas, we do not detect a difference in HP content in lung homogenates between P-2119 and saline in short term studies (3 weeks after bleomycin), persistent HP increases are observed in SFTPC-Muc5 $\mathrm{b}^{\mathrm{Tg}}$ mice over a longer period (10 weeks) and these changes are significantly reduced by P-2119 treatment (Fig. 4c). Importantly, histologically detectable fibrillar collagen detected by SHG imaging decreases in the lungs of SFTPC-Muc $5 b^{T g}$ mice treated with P-2119, and this is observed in both the 3 week and 10 week model of bleomycin-induced lung fibrosis (Fig. $4 \mathrm{~d}-\mathrm{h}$ ). Strikingly, in the absence of P-2119 treatment, HP values increase in SFTPCMuc $5 b^{\mathrm{Tg}}$ mice during prolonged responses to bleomycin (Fig. 4c). However, the concentration of fibrillar collagen decreases over time (Fig. 4d). This discrepancy could reflect differences in the types of collagen detected since unlike SHG, HP does not distinguish fibrillar vs. non-fibrillar types of collagen. Also, SHG image analyses did not include central airways and vasculature, but were instead conducted on peripheral lung tissues (the location of pulmonary fibrosis in humans). Importantly though, for both HP and SHG analyses, P-2119 significantly reduces acute and protracted lung fibrosis. Taken together, these findings highlight the importance of short and long-term models of lung fibrosis and comprehensive assessment of outcomes, including secondary endpoints, such as survival, along with the critical primary endpoint of fibrosis in parenchymal lung tissues.

\section{Discussion}

Mucociliary dysfunction is an emerging paradigm in lung diseases 19,20 . Previously considered a characteristic specific to obstructive diseases such as asthma and chronic obstructive pulmonary disease, and genetic diseases such as primary ciliary dyskinesia and cystic fibrosis, the importance of mucins, mucus, and mucociliary interactions has surfaced in diseases of the lung periphery, such as adenocarcinoma and $\mathrm{IPF}^{4,5}$. Unlike obstructive diseases, which involve central conducting airways and rapid defense against ubiquitous environmental challenges, mucus dysfunction in the lung periphery appears to involve injury-repair processes predominantly $5,10,21$. In this vein, infection, mucous metaplasia, and bronchiectasis that occur with mucociliary dysfunction in conducting airways contrast with pathologic changes, such as bronchioloalveolar epithelial and mesenchymal remodeling or proliferation that occur with mucin overexpression in the lung periphery ${ }^{21-26}$.

Our results demonstrate that elevated concentrations of Muc5b in the distal lung are directly related to the fibroproliferative response to bleomycin in mice, and MCC dysfunction might play an important role in this response. Previous findings in knockout mice showed MCC failure in the absence of Muc5b $b^{10}$, so dysfunctional MCC due to high concentrations of Muc5b may seem counterintuitive. However, the distribution of mucous and ciliated cells, the anatomical location of $M U C 5 B / M u c 5 b$ expression, and the homeostatic control of mucus hydration are factors that a
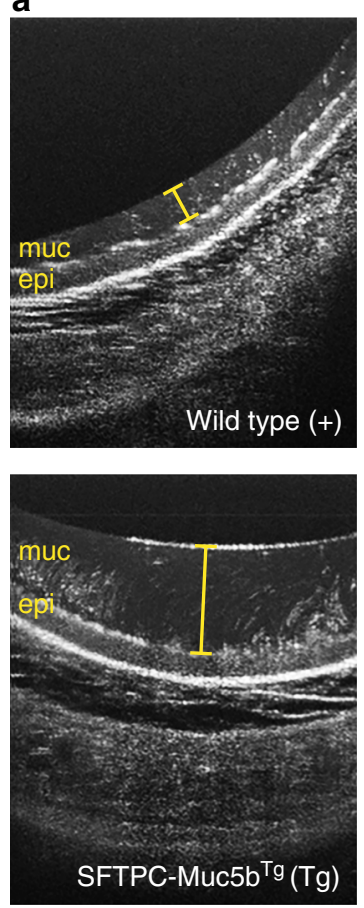

b

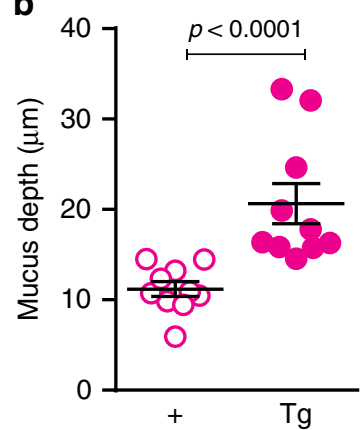

d

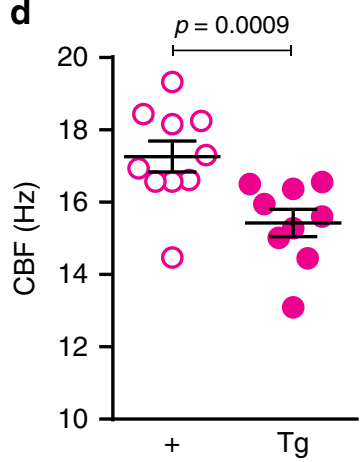

C

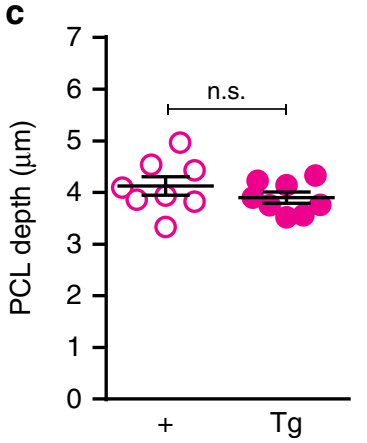

e

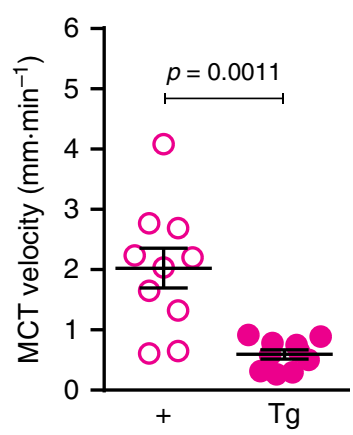

Fig. 2 Muc5b overexpression in SFTPC-Muc5bTg mice is associated with impaired mucociliary transport. a Representative $\mu$ OCT images of excised SFTPCMuc5bTg mice trachea in comparison to trachea of wild type (+) littermate controls. b-e Quantitative metrics from image analysis reveal increased mucus layer depth $\mathbf{b}$ without significant alteration of periciliary layer (PCL) depth $\mathbf{c}$ in $\mathrm{Tg}$ mice compared to + controls. Functional analysis demonstrated reduced ciliary beat frequency $\mathbf{d}$ and diminished mucociliary transport rates $\mathbf{e}$ in Muc5b-overexpressing mice. Data in $\mathbf{b}$-e are means \pm sem and were analyzed by Mann-Whitney test 
a

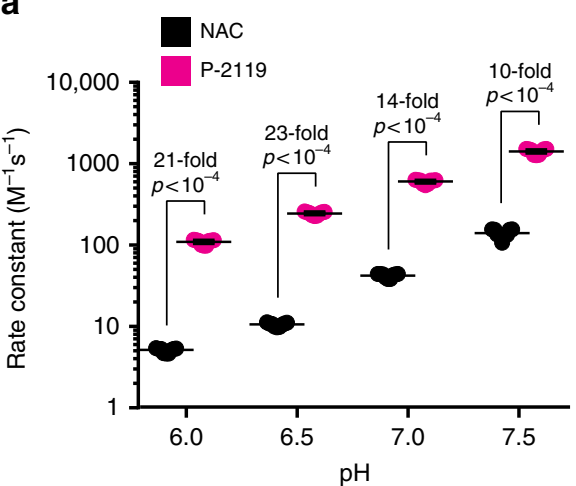

b

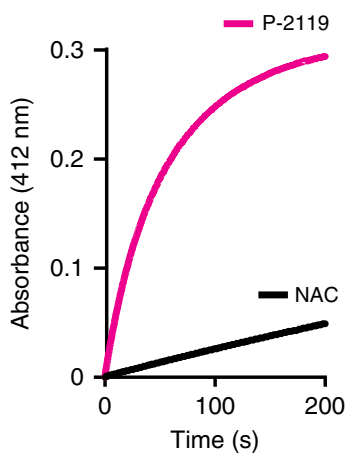

c

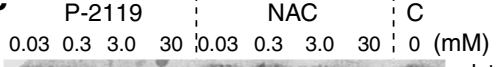

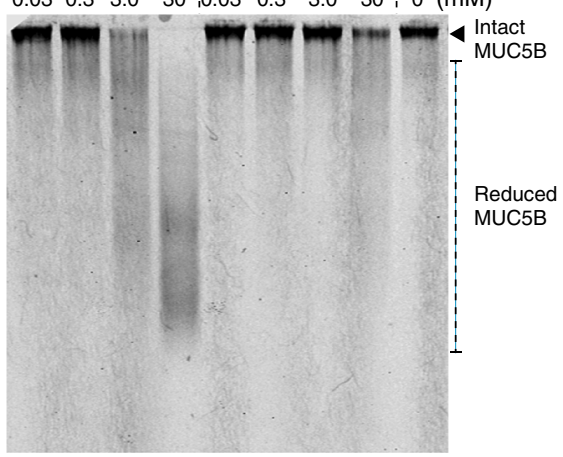

d

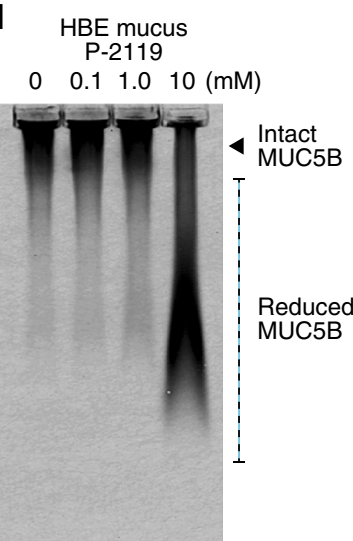

h

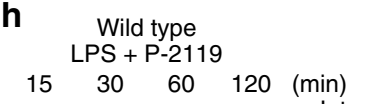

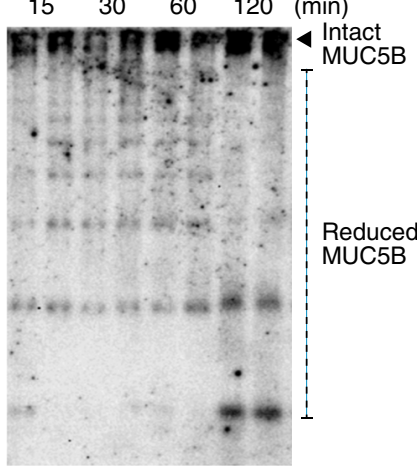

e
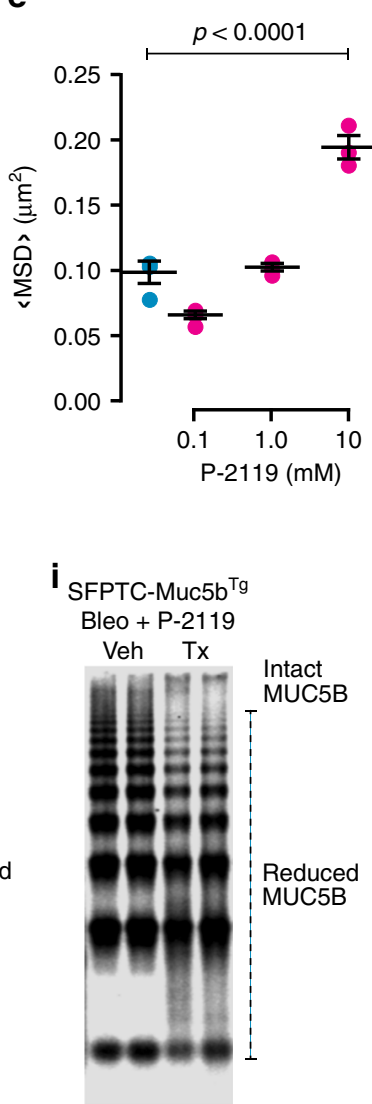

f

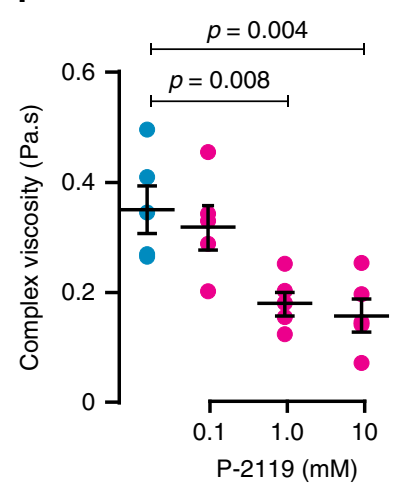

g

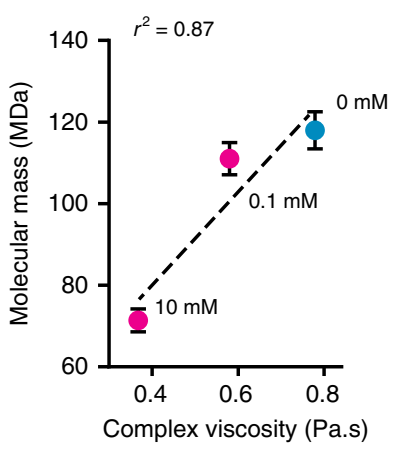

j

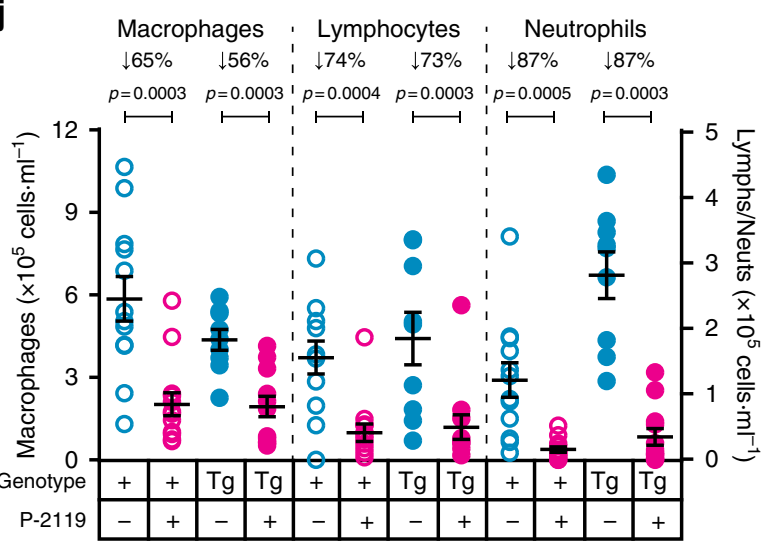

Fig. 3 P-2119 effectively cleaves mucus in vitro and in vivo, enhancing the acute clearance of inflammatory cells. a, b P-2119 hydrolyzed DTNB disulfide bonds more quickly than $\mathrm{n}$-acetylcysteine (NAC) $\mathbf{a}$, and at pH 6 P-2119 cleaved more bonds than NAC $\mathbf{b}$. $\mathbf{c}$ In human saliva, P-2119 reduced MUC5B in salivary mucus at lower concentrations than NAC. $\mathbf{d}-\mathbf{g}$ In concentrated normal human bronchial epithelial cell culture mucus (5\% solids), P-2119 reduced MUC5B at a potency similar to that seen in saliva in c. Reduction of MUC5B by P-2119 dose dependently lowered mucus viscosity as demonstrated by enhanced mean square displacement (MSD) of fluorescent microspheres $\mathbf{e}$, and as shown by improved macrorheological complex viscosity $\mathbf{f}$ that was strongly correlated with reduced molecular mass $\mathbf{g}$. Data in e represent 900 technical and three biological replicates; data in $\mathbf{f}$ represent three technical and two biological replicates. Cyan symbols, vehicle; magenta symbols, P-2119. $\mathbf{h}-\mathbf{j}$ In vivo effects of aerosolized P-2119 (68-135 mM for $60 \mathrm{~min}$ ). h Wild-type mice challenged with LPS $(20 \mu \mathrm{g}, \mathrm{IT}) 48 \mathrm{~h}$ prior to P-2119 aerosol. P-2119 decreased Muc5b mass detected by immunoblot of lung lavage fluid over a 120 min period. i SFTPC-Muc5 $\mathrm{b}^{\mathrm{Tg}}$ mice were challenged with bleomycin $(2.5 \mathrm{U} / \mathrm{kg}$, IT) $7 \mathrm{~d}$ prior to P-2119 treatment (Tx). P-2119 caused Muc5b reduction detected by immunoblot 120 min post initiation of P-2119 aerosol. $\mathbf{j}$ The effect of P-2119-induced mucolysis on MCC was assessed by quantifying the acute elimination of leukocytes in lung lavage fluid obtained from bleomycin treated SFTPC-Muc5b ${ }^{\top g}$ mice $(n=9$ vehicle and 12 P-2119 treated) and wild type $(+)$ controls ( $n=12$ vehicle and 13 P-2119 treated). Total cells were significantly lower in P-2119 treated mice compared to vehicle treated animals, reflecting decreases in all leukocyte subtypes in bleomycin-injured lungs. Data in $\mathbf{a}, \mathbf{e}-\mathbf{g}$, and $\mathbf{j}$ are means \pm sem. Data in $\mathbf{a}$ were analyzed between P-2119 and NAC treated groups using $t$-tests. Data in $\mathbf{e - g}$ were analyzed statistically on biological replicates: ANOVA of results between $0 \mathrm{mM}$ vehicle and 0.1-10 mM P-2119 treatment groups $\mathbf{e}, \mathbf{f}$ and linear regression of complex viscosity vs mass $\mathbf{g}$ 

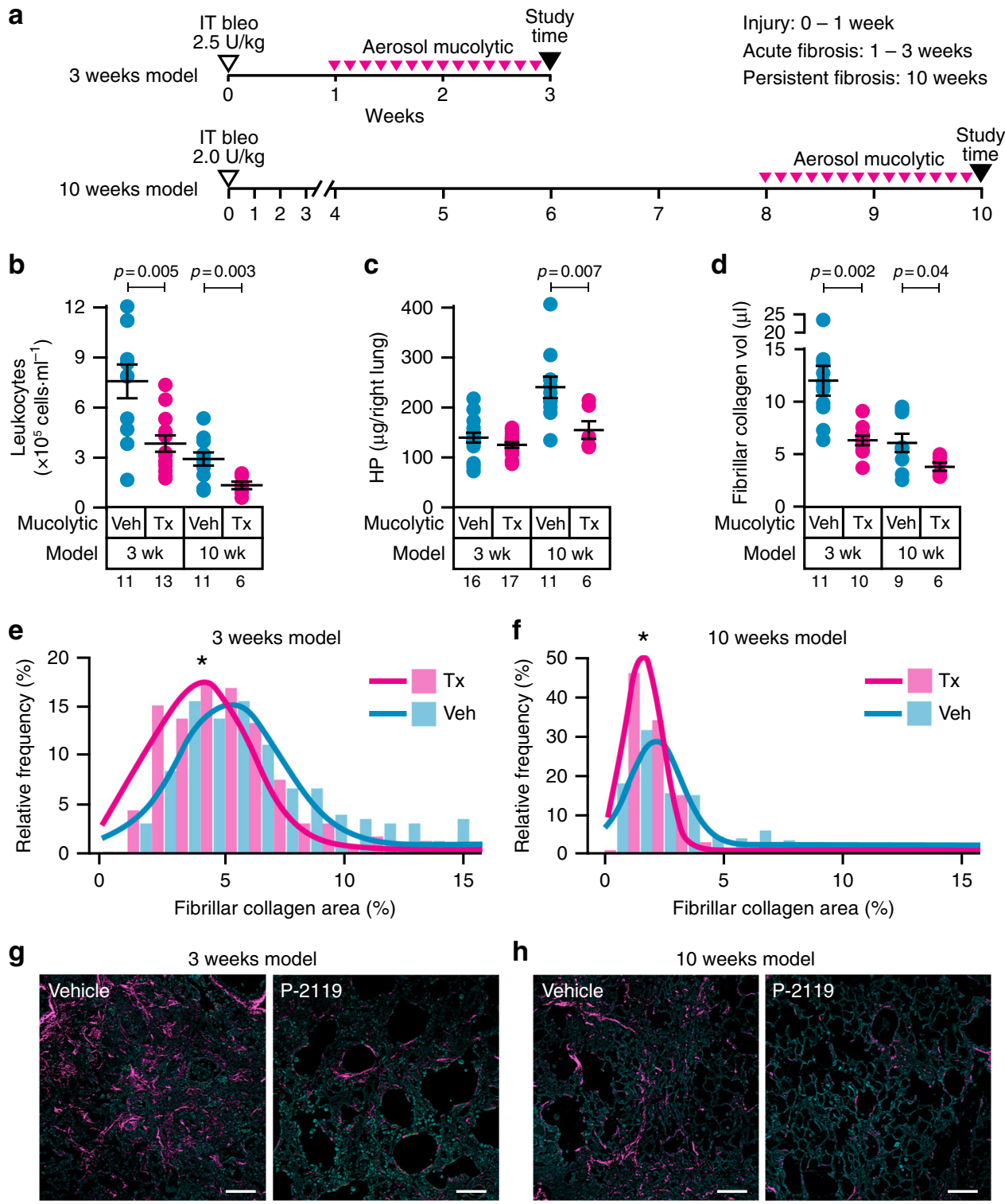

h
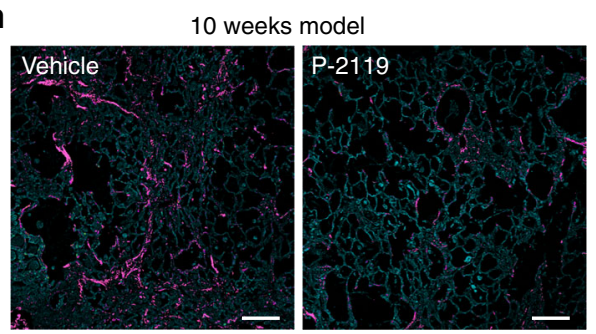

Fig. 4 P-2119 treatment results in reduced collagen deposition in the setting of excess Muc5b. a SFTPC-Muc5bTg mice were subjected to bleomycin challenge with a 2 wk P-2119 intervention protocol, with daily P-2119 treatments (Tx) or saline vehicle (Veh) exposures starting 7 or $56 \mathrm{~d}$ post bleomycin challenge and ending $24 \mathrm{~h}$ prior to tissue harvest. $\mathbf{b}$-h Injury and fibrosis induced in bleomycin-treated mice exposed to saline vehicle were decreased in P2119 treated animals. Compared to vehicle treated animals, P-2119 treated mice had significantly fewer leukocytes in lung lavage $\mathbf{b}$, less hydroxyproline in lung homogenates at 10 wks $\mathbf{c}$, and decreased peripheral lung fibrillar collagen $\mathbf{d}-\mathbf{h}$. In $\mathbf{b}-\mathbf{d}$, data are means \pm sem, numbers in italics indicate $n$ animals used per experiment, and $p$-values indicate differences determined by $t$-test with Welch's correction for unequal variances. In $\mathbf{e}$ and $\mathbf{f},{ }^{*}$ indicates statistical significance by $t$-test $(p<0.00001)$. Histograms in $\mathbf{e}$ are from 15 Veh mice (225 images) and from $12 \mathrm{Tx}$ mice (165 images); histograms in $\mathbf{f}$ are from 9 Veh mice (180 images) and from 6 Tx mice (120 images). Images in $\mathbf{g}$ and $\mathbf{h}$ show greater amounts of fibrillar collagen (red) in Veh vs. Tx groups. Scale bars, $100 \mu \mathrm{m} \mathbf{g}, \mathbf{h}$

coordinately affect mucus viscoelasticity and transport ${ }^{5}$. Accordingly, ectopic overproduction of Muc5b in the lung periphery in SFTPC-Muc5b ${ }^{\text {Tg }}$ mice (see Fig. $1 c$ and Supplementary Fig. 1c) produces more severely defective mucociliary transport than what is observed in Scgbla1-Muc5b $b^{\mathrm{Tg}}$ mice (see Fig. 2e and Supplementary Fig. 2e), where Muc5b overproduced by club cells in airways where it is also normally expressed ${ }^{27-29}$. These findings suggest that overexpression of $M U C 5 B$ in distal airspaces, which is known to occur in $\mathrm{IPF}^{4,9}$, disrupts the equilibrium necessary to sustain effective mucociliary transport ${ }^{13,30}$ thereby impairing mucus function ${ }^{12,26,31}$.

One potential consequence of mucociliary dysfunction is retention of inhaled substances (air pollutants, cigarette smoke, microorganisms, etc.) and endogenous inflammatory debris that, over time, results in temporally and spatially distinct areas of microscopic scaring and progressive fibroproliferation in the lung leading to the development of $\mathrm{IPF}^{1}$. Alternatively, reduced clearance or enhanced viscosity of MUC5B may initiate a reactive or regenerative fibrotic response localized to the bronchoalveolar region of the lung that eventually leads to the development of IPF. As we observed that MUC5B is co-expressed with surfactant protein $\mathrm{C}$ in type 2 alveolar epithelia and cells lining honeycomb cysts in human IPF (Fig. 1a, b), we postulate that the cells involved in MUC5B overexpression are involved in the lung remodeling that is characteristic of IPF.

Whereas there is no true equivalent animal model of IPF, intratracheal bleomycin challenge is a widely used model of lung fibrosis in mice. Nonetheless, both bleomycin challenge as a 
trigger and the mouse as a species have strengths and weaknesses ${ }^{32}$. Bleomycin causes an acute increase in collagen production that can resolve over time, but bleomycin can induce prolonged lung fibrosis under some conditions, including with repeated dosing ${ }^{11,33}$ as we have done here (Fig. 1c-i). In addition, mouse lungs are anatomically different from humans. Mice lack respiratory bronchioles and demonstrate little or no mucin expression in the lung periphery ${ }^{27-29}$. By using SFPTC-Muc5b $\mathrm{b}^{\mathrm{Tg}}$ mice, we help to significantly narrow this species gap. Importantly, SFTPC-Muc5bTg mice developed prolonged fibrosis in a single bleomycin challenge setting (Fig. 4). Thus, even in an acute injury setting that differs from the chronic development of disease in humans, this Muc5b-overexpressing model provides useful insight in an IPF-related setting. Other aspects such as microscopic honeycombing and proliferative repair vs. remodeling need to be explored ${ }^{11,33}$. The last has been implicated with much of the focus on expansion of fibroblasts and alveolar remodeling suggestive of a process of active disease progression. Our findings support a role for mucociliary dysfunction in active disease, and they support a long-term goal in the field of identifying ways to slow or reverse the development of IPF at early and/or preclinical stages.

The potential role for mucociliary dysfunction as a driver of IPF pathology is supported by unique gene expression signatures in IPF. In patients with IPF, the coordinated overexpression of $M U C 5 B$ and cilium genes is associated with microscopic honeycombing, a pathognomonic feature of $\mathrm{IPF}^{34}$. Furthermore, cilium and $M U C 5 B$ gene expression are associated with the concentration of $M M P 7$, a metalloproteinase gene that is a known biomarker in IPF $^{35}$ and is also known to amplify airway ciliated cell differentiation ${ }^{36}$. Adequate MCC requires a balance between the concentrations of water, ions, and macromolecules in the mucus gel. Excessive production of MUC5B presents a challenge to proper mucus hydration and cilia function ${ }^{12}$. In equilibrium, airway surface liquid homeostasis maintains a low-friction state within the periciliary layer (PCL). In diseases such as cystic fibrosis, a strong transcellular osmotic gradient causes hyperabsorption of ions and water by airway epithelial cells, generating mucus dehydration, reduced cilia motility, and in extreme cases cilia collapse. This phenomenon can also be caused by the presence of high concentrations of mucins on the apical surfaces of airways, generating similar dehydrating forces as observed in CF; excessive mucin could result in an osmotic gradient favoring water movement out of the PCL and towards airway lumen ${ }^{12,37}$. In both cases, the loss of a grafted gel-on-brush confirmation results in excessive mucus aggregation and impaired MCC. Finally, when mucin expression is uncoupled with CFTRdependent anion secretion, abnormally viscous mucus could ensue beyond the effects of airway dehydration, precipitating abnormal host defense ${ }^{15}$. Whereas the findings here do not support PCL depletion as a mechanism for mucociliary dysfunction in Muc5b-overproducing mice at baseline (see Fig. 2c and Supplementary Fig. 2c), it is possible that PCL depletion may be detectable in injured mice. Additional studies will identify how these biophysical properties are regulated and alter mucociliary function, as well as the extent to which genetic factors such as the MUC5B promoter variant rs35705950 impact airway epithelial cell biology.

The relationship between MUC5B overproduction and IPF is complex. Although Muc5b transgenic mice do not appear to spontaneously develop pulmonary fibrosis, they are more responsive to bleomycin (Fig. 1f-i). Likewise, the human gain-offunction $M U C 5 B$ promoter variant that causes overexpression of $M U C 5 B$ in the distal airspace also does not appear to be sufficient to cause pulmonary fibrosis. Although $\sim 20 \%$ of non-Hispanic Whites have at least one copy of the MUC5B promoter variant ${ }^{4}$, IPF is a rare disease occurring in less than $0.1 \%$ of the population $^{1}$. Despite the caveat that IPF is likely underdiagnosed ${ }^{38-40}$, it remains clear that the $M U C 5 B$ promoter variant represents a low penetrance allele. It is thus likely that other gene variants and/or environmental exposures interact with the $M U C 5 B$ promoter polymorphism to cause IPF in individuals with this diseaseassociated genetic variant. These observations lead us to postulate that the etiology of IPF will best be understood by identifying the genes, transcripts, and environmental exposures that interact with MUC5B and contribute to the development of IPF. While future studies in large populations of patients with IPF may reveal the critical biological mechanisms that interact with the gain-offunction MUC5B promoter variant to cause IPF, our Muc5b transgenic mice may prove critically important in identifying the genes and/or environmental exposures that contribute to this complex disease.

In a broader context, these findings suggest that by identifying those at risk, patients could be diagnosed prior to the development of permanent and extensive lung parenchymal scarring. Genetic risk factors, particularly the MUC5B promoter variant ${ }^{4}$, have been shown to identify individuals with preclinical interstitial changes on chest CT $\operatorname{scan}^{38-40}$ that progress to clinical significance and are associated with reduced survival ${ }^{39}$. Given the irreversible nature of IPF, even approved treatments (pirfenidone $^{2}$ and nintedanib ${ }^{3}$ ) only modestly slow progression and have not been shown to alter the median 3-5 year survival. Patients with preclinical stages of pulmonary fibrosis may be ideal candidates for early intervention focused on avoiding the development of irreversible lung remodeling. Our findings suggest that targeting MUC5B in the terminal airways of patients with preclinical stages of interstitial lung disease represents a rational strategy to prevent the progression of preclinical pulmonary fibrosis.

\section{Methods}

Human lung specimens. Lung specimens from patients with idiopathic pulmonary fibrosis (IPF) and unaffected controls were obtained from the NHLBI Lung Tissue Research Consortium (LTRC; https://ltrcpublic.com/). All protocols were performed in compliance with all relevant ethical regulations approved by local Institutional Review Boards, and individuals gave written informed consent to participate.

Mouse husbandry. Studies using animals complied with all relevant ethical regulations. Mice were housed in accordance with the Institutional Animal Care and Use Committee of the University of Colorado and University of Alabama at Birmingham and kept in specific-pathogen free housing areas that were monitored by institutional animal care staff. Mice were maintained on a $12 \mathrm{~h}$ light/dark cycle and fed ad libitum a normal diet of water and irradiated chow (Harlan Teklad). Mice were assigned non-descriptive numbers at weaning by attachment of an ear tag. Moribund mice were identified by observing changes in body weight and behavior. In accordance with the veterinary care procedures at University of Colorado, a loss of $15 \%$ of body weight without recovery, hunching, fur ruffling and lethargy were used as criteria for determining moribundity. Animals were killed by intraperitoneal injection of sodium pentobarbital followed by exsanguination.

Genetically engineered mice. Muc $5 b^{+/-}$mice were generated previously ${ }^{10}$, and heterozygous males and females were bred to obtain male Muc5 $\mathrm{b}^{-/-}$and Muc5 $\mathrm{b}^{+/+}$ littermates for experiments. Scgblal-Muc5 $\mathrm{b}^{\mathrm{Tg}}$ mice were generated previously ${ }^{1}$ and were maintained by continuous hemizygous outcrosses with C57BL/6J male mice purchased from the Jackson Laboratory. SFTPC-Muc $5 b^{T g}$ mice were created by insertion of the full-length $34-\mathrm{kb}$ genomic coding region of the mouse $\mathrm{Muc5}$ b gene into a transgenic targeting cassette containing $3.7 \mathrm{~kb}$ of the human SFTPC gene $5^{\prime}$ flanking region and an IRES-mCherry reporter. Founders were generated by injecting the targeting vector into $\mathrm{C} 57 \mathrm{BL} / 6 \mathrm{~N}$ pronuclei at the National Jewish Health transgenic mouse core. After confirmation of Muc5b overexpression by western blot, SFTPC-Muc $5 b^{T g}$ mice were maintained by continuous hemizygous outcrosses with C57BL/6J male mice purchased from the Jackson Laboratory.

Bleomycin exposure. All mice began bleomycin treatment between 8 and 12 weeks of age, and only male mice were used due to their known sensitivity to bleomycin ${ }^{41,42}$. Mice were anesthetized with inhalational isoflurane (MWI Veterinary Supply Company, Boise, ID) and tracheas were directly visualized with a rodent laryngoscope (Penn Century, Wyndmoor, PA). A 22 g gavage needle was used to instill $50 \mu \mathrm{l}$ of saline or bleomycin solution (APP Pharmaceuticals, Schaumburg, IL) directly into the trachea. Studies were conducted following repeated bleomycin challenges (Fig. 1) or following a single bleomycin challenge (Figs. 3, 4 and Supplementary Fig. 4). For repeated bleomycin challenge studies 
shown in Fig. 1d, e, animals received $2.5 \mathrm{U} / \mathrm{kg}$ bleomycin in d 0, followed by $1.5 \mathrm{U} /$ $\mathrm{kg}$ on $\mathrm{d} 14$ and $\mathrm{d} 28$. For studies shown in Fig. $1 \mathrm{f}-\mathrm{i}$, SFTPC-Muc $5 \mathrm{~b}^{\mathrm{Tg}}$ mice received $2.0 \mathrm{U} / \mathrm{kg}$ on $\mathrm{d} 0$, followed by $1.0 \mathrm{U} / \mathrm{kg}$ on $\mathrm{d} 14$ and $\mathrm{d} 28$. All repeat challenge animals were collected on d 49 . For single bleomycin challenge studies, SFTPCMuc5 $\mathrm{b}^{\mathrm{Tg}}$, mice received $2.5 \mathrm{U} / \mathrm{kg}$ on d 0 and were studied on $\mathrm{d} 7$ (Fig. 3), d 21 (Fig. 4), or d 70 (Fig. 4).

Bronchoalveolar lavage. Immediately following euthanasia, mouse tracheas were cannulated and the lungs lavaged three times with $0.5 \mathrm{ml}$ of PBS containing 0.6 mM EDTA. Cells were counted using a hemacytometer and spun on to slides using a Cytospin 4 (Thermo Fisher Scientific, Waltham, MA). The slides were stained with the Hema 3 kit (Thermo Fisher) and used for differential counts of macrophages, lymphocytes, and neutrophils. Remaining lavage fluid was divided into two aliquots. One portion was centrifuged at $300 \times \mathrm{g}$ for $10 \mathrm{~min}$ at $4^{\circ} \mathrm{C}$ for subsequent studies of cytokines and other soluble factors, and the other portion was stored without centrifugation to preserve high molecular weight mucins that sediment at low centrifugation speeds. Both were frozen on dry ice and stored at $-80^{\circ} \mathrm{C}$.

Hydroxyproline. For repeat bleomycin studies, the entire right lung was removed, added to $550 \mu \mathrm{l}$ PBS and homogenized using Lysing Matrix D and a FastPrep-24 bead beater (MP Biomedicals, Santa Ana, CA). Samples were then snap frozen and stored at $-80^{\circ} \mathrm{C}$. Thawed lung homogenates were hydrolyzed overnight with a 1:1 volume of $12 \mathrm{~N} \mathrm{HCl}$ at $100^{\circ} \mathrm{C}$. Afterwards, $5 \mu$ samples of hydrolyzed lung and hydroxyproline standards were plated in duplicate in 96-well plates and incubated for $20 \mathrm{~min}$ in $100 \mu \mathrm{l}$ of $0.06 \mathrm{M}$ chloramine T in citrate-acetate buffer, $\mathrm{pH}$ 6. Ehrlich's solution ( $100 \mu \mathrm{l} ; 1.2 \mathrm{M}$ dimethylaminobenzaldehyde in $22 \%$ perchloric acid$n$-propanol) was then added to each sample. After a 20 -min incubation at $65^{\circ} \mathrm{C}$, plates were analyzed in an Synergy H1 plate reader (Biotek, Winooski, VT) at 550 $\mathrm{nm}$ for colorimetric analysis. Concentrations of each sample were determined by interpolation along a standard curve.

For P-2119 studies $^{17}$, the right upper, lower, and accessory lobes were homogenized in $500 \mu \mathrm{l}$ PBS and then processed as above.

Histochemistry and immunohistochemistry. At harvest, the left lung was inflated with $4 \%$ paraformaldehyde (PFA) at a pressure of $20 \mathrm{~cm} \mathrm{H}_{2} \mathrm{O}$ for $5 \mathrm{~min}$. The lung was then removed and placed in fresh 4\% PFA overnight for fixation. Left lungs were cut into uniform slices, and volumes were recorded using Cavalieri imaging calculations. Lungs were then embedded in paraffin, cut into $5 \mu \mathrm{m}$ sections, and collected on positively charged glass slides. For hematoxylin and eosin staining, tissues were stained using standard reagents at the University of Colorado Cancer Center Pathology Core.

For immunohistochemistry, tissues were heated in citrate buffer for antigen retrieval (20 min boiling), and tissues were then incubated overnight in goat primary antisera against Muc5b (1:1000, Everest Biotech, Upper Heyford, UK) or with rabbit primary anti-mouse Muc5b antisera $(1: 20,000)^{10}$. Secondary anti-goat antibody diluted 1:1000 tagged with AlexaFluor488 (Thermo Fisher) or ImmPRESS anti-rabbit conjugated with horseradish peroxidase (Vector Laboratories) were applied for $1 \mathrm{~h}$ at room temperature. Immunofluorescence slides were coverslipped with VECTASHIELD Hardest mounting medium with DAPI (Vector). DAB stained slides were counterstained with hematoxylin and permanently mounted. Samples were visualized using an Olympus BX63 microscope (Olympus, Tokyo, Japan).

In situ hybridization. RNAScope detection was used to perform in situ hybridization according the manufacturer's protocol (Advanced Cell Diagnostics, Hayward, CA). Briefly, formalin-fixed paraffin embedded human lungs were cut into 5 $\mu \mathrm{m}$ thick tissue sections. Slides were deparaffinized in xylene, followed by rehydration in a series of ethanol/water washes. Following citrate buffer (Advanced Cell Diagnostics) antigen retrieval, slides were rinsed in deionized water, and immediately treated with protease (Advanced Cell Diagnostics) at $40^{\circ} \mathrm{C}$ for $30 \mathrm{~min}$ in a HybEZ hybridization oven (Advanced Cell Diagnostics). Probes directed against $M U C 5 B$ and SFTPC mRNA and control probes were applied at $40^{\circ} \mathrm{C}$ in the following order: target probes, preamplifier, amplifier; and label probe for $15 \mathrm{~min}$. After each hybridization step, slides were washed two times at room temperature. Chromogenic detection was performed followed by counterstaining with hematoxylin (American MasterTech Scientific, Lodi, CA). Staining was visualized using an Olympus BX63 microscope using a $\times 60$ oil immersion lens, $z$-stack imaging, extended focal image processing (Olympus, Tokyo, Japan).

Second harmonic generation (SHG). Autofluorescence and SHG signals were acquired using Zeiss 780 laser-scanning confocal/multiphoton-excitation fluorescence microscope with a 34-channel GaAsP QUASAR detection unit and nondescanned detectors for two-photon fluorescence (Zeiss, Thornwood, NY). The imaging settings were initially defined empirically to maximize the signal-to-noise ratio and to avoid saturation; and they were kept constant for all measurements for comparative imaging and results. Seven percent of a two-photon Chameleon laser tuned to $800 \mathrm{~nm}$ was used for excitation, and emission signals corresponding to the autofluorescence and SHG signals were detected simultaneously through nondescanned detectors. Image processing was performed using Zeiss ZEN
2012 software. Fifteen images were obtained for each of the lung using standardized uniform random sampling ${ }^{43}$. The series of images were analyzed in Image J software. Percentage of area covered by fibrillar collagens was quantified for each slide, and collagen fractions were normalized to lung volumes. Histograms for cumulative data for each group was created using GraphPad Prism (GraphPad, La Jolla, CA).

Micro-optical coherence tomography. Adult SFTPC-Muc5b ${ }^{\mathrm{Tg}}$ and Scgbla1Muc $5 b^{T g}$ mice at 8-12 weeks old and their age-matched littermate were killed using ketamine and xylazine. Tracheas were removed, separated from the distal airways and lung tissue, and placed in HEPES-buffered DMEM. Tracheal tissue was then dissected along the transverse axis to expose the luminal epithelial cell surface and incubated for $30 \mathrm{~min}$ under physiologic conditions $\left(37^{\circ} \mathrm{C}, 5 \% \mathrm{CO}_{2}\right.$, $100 \%$ humidity) to allow the epithelial surface to equilibrate.

Functional assessments of the tracheal tissue explants were performed using $\mu \mathrm{OCT}$, with acquisition speed set at $20,480 \mathrm{~Hz}$ line rate to yield 100 frames per second at 256 lines per frame. Images were recorded in 8-10 ROI per trachea by an imaging specialist blinded to genotype. Images were recorded at randomly chosen intervals on the mucosal surface with the optical beam scanned along the longitudinal direction ${ }^{16,44}$.

Several metrics were simultaneously quantified from $\mu$ OCT-recorded images using Image J and Matlab. Airway surface liquid (ASL) and Periciliary layer (PCL) depths were measured directly ${ }^{44}$. Mucociliary transport rate was calculated based on the slope of mucus particulates in the mucus over several frames in the ASL region up to $50 \mu \mathrm{m}$ above the epithelial cell surface. CBF was determined using Fourier transform analysis of the reflectance of beating cilia. Imaging analysis was performed in a blinded fashion with respect to genotype.

In vitro mucolytic testing. The rate of thiol-disulfide exchange was assayed for NAC and P-2119 utilizing Ellman's reagent (5,5'-dithiobis-2-nitrobenzoic acid or DTNB) and methods adapted by Han and Han ${ }^{45}$. Briefly, NAC or P-2119 was added to a final concentration of $22.5 \mu \mathrm{M}$ in an excess of DTNB $(45 \mu \mathrm{M})$ and formation of the fluorescent DTNB cleavage product (2-nitro-5-thiobenzoate) was monitored over time at $412 \mathrm{~nm}$ by a spectrophotometer.

MUC5B was analyzed using whole saliva or mucus purified from primary human bronchial epithelial cell cultures and concentrated to $5 \%$ solids $(\mathrm{w} / \mathrm{v})^{46}$. Briefly, mucus samples were treated with reducing agents NAC or P-2119 (0.03-30 $\mathrm{mM}$ ) for $1 \mathrm{~h}$ at room temperature. Mucin polymer reduction was assessed by agarose-gel western blot (see Supplementary Fig. 8 for uncropped scans). The mucus samples were alkylated with $\mathrm{N}$-ethylmaleamide $(100 \mathrm{mM}$ final concentration) and mucins were separated by $1 \%(\mathrm{w} / \mathrm{v})$ agarose-gel electrophoresis vacuum-blotted onto a PVDF membrane, and detected by western blotting with MUC5B antibodies ${ }^{47}$.

Mucus microrheology. Microbead rheology was performed by tracking the thermally driven motion of embedded $1 \mu \mathrm{m}$ diameter carboxylated microspheres (FluoSpheres, Fischer Scientific) $^{37,48,49}$. Briefly, microspheres were added to mucus and allowed to mix while rotating overnight at $4{ }^{\circ} \mathrm{C}$. After reduction, fifteen $30 \mathrm{~s}$ movies were collect at 60 frames per second on a Nikon Eclipse TE 2000 microscope at $\times 40$ with a Flea 3 camera (FLIR Machine Vision, Richmond BC, Canada). Particle trajectories were subsequently tracked using TrackPy (v2.4, https://doi.org/ 10.5281/zenodo.12255). The track positions were corrected in Matlab (The MathWorks, Natick, MA) to account for linear drift and mean squared displacement (MSD) was calculated for each bead according to Equation 1

$$
\Delta r^{2}(\tau)=\frac{1}{N-\tau} \sum_{i=1}^{N-\tau}\left(x\left(t_{i}+\tau\right)-x\left(t_{i}\right)\right)^{2}+\left(y\left(t_{i}+\tau\right)-y\left(t_{i}\right)\right)^{2} \#,
$$

where $N=1800$ total frames and $\tau$ is the time-lag.

Mucus macrorheology. Mucus was collected from human bronchial epithelium (HBE) cell culture models ${ }^{48,50,51}$ and prepared to $5 \%$ solids to mimic the concentration seen in obstructive airways disease. Mucus was treated with concentrations of compound from $0.1 \mathrm{mM}$ to $10 \mathrm{mM}$ and allowed to incubate for $1 \mathrm{~h}$ at $37^{\circ} \mathrm{C}$. The rheological properties are determined by analyzing the linear regime of a fixed frequency stress sweep ${ }^{50,51}$. All assays were performed on a Discovery Hybrid 3 rheometer with $20 \mathrm{~mm}$ diameter 10 cone with solvent trap (TA Instruments, New Castle Delaware). Data were analyzed using custom Matlab scripts (MathWorks). All treatments we performed by mixing P-2119 in PBS at pH 7 to 100 -fold higher than the desired final concentration, and $10 \mu \mathrm{l}$ of compound was added to $90 \mu \mathrm{l}$ of $\mathrm{HBE}$ mucus and incubated at $37^{\circ} \mathrm{C}$ for $1 \mathrm{~h}$. All assays were performed at $23^{\circ} \mathrm{C}$ to prevent evaporation.

Multi-angle laser light scattering spectrometry. The molecular weight determination is performed on a aliquot of the same mucus that was used for rheology. Ten microliters of mucus is added to $40 \mu \mathrm{l}$ of $6 \mathrm{M}$ guanidinium HCl. Samples were then further diluted 1:20 into light scattering buffer for high-pressure liquid chromatography (HPLC) on a CL2B column to separate large molecular weight mucins from other small proteins. HPLC was run in combination with 
refractometry (TRex, Wyatt) and multi-angle laser light scattering (MALLS, DAWN Heleos II, Wyatt Technologies) to determine molecular weight (shown) and mucin concentration (unchanged by reduction).

Aerosol exposure. Solutions were nebulized with an Aeroneb nebulizer. Aerosolized P-211917 was delivered to a 24-port nose-only inhalation chamber (In-Tox Products, Inc., Albuquerque, NM) operated at $~ 10$ l/min. Exposure monitoring was conducted by collection of air samples from the test atmosphere on to $47 \mathrm{~mm}$ Teflon membrane filters (TEFLO, Pall-Gelman) that were weighed before and after sample collection. The aerosol was withdrawn directly from the exposure chamber atmosphere with a flow rate of $\sim 1$ liter/minute. Particle size was $\sim 1 \mu \mathrm{m}$ with a geometric standard deviation of $\sim 2.0$.

In vivo mucolytic testing. To test mucolytic activity, mice were tested under two conditions. First, wild-type C57BL/6J mice were challenged IT with $20 \mu \mathrm{g}$ of LPS (E. coli 055:B5) administered in a $50 \mu \mathrm{l}$ volume of saline. Mice were then treated $2 \mathrm{~d}$ post LPS challenge with P-2119 $(135 \mathrm{mM})$ or vehicle (normal saline) for $60 \mathrm{~min}$. Mice were studied 15, 30, 60, and $120 \mathrm{~min}$ post P-2119 treatment (68 mM aerosol) to evaluate the potency and kinetics of mucolysis induced by P-2119. Second, a separate group of SFTPC-Muc5 $5 \mathrm{~b}^{\mathrm{Tg}}$ mice was then analyzed following a single IT bleomycin challenge. Bleomycin treated mice were then treated with P-2119 (135 $\mathrm{mM}$ ) or vehicle (normal saline) by aerosol for $60 \mathrm{~min}$ on $\mathrm{d} 7$ post challenge, a peak point of bleomycin-induced inflammation. Mice were studied immediately after withdrawal from P-2119 or vehicle challenge or $1 \mathrm{~h}$ after P-2119 (60 and $120 \mathrm{~min}$ timepoints post initiation of treatments, respectively). Mucolysis was assessed in un-centrifuged lung lavage fluid that was treated with $1 \mathrm{M}$ iodoacetamide to quench drug activity and alkylate thiols liberated by disulfide reduction. Western blotting was performed as described above for human MUC5B, in this case with rabbit-anti-mouse Muc5b antisera.

We further evaluated the functional consequences of mucolytic treatments mouse lungs. The same LPS (d 2), bleomycin (d 7), and mucolytic treatment groups described above for assessing mucolysis biochemically were used to assess mucolytic effects on MCC and mucus obstruction. To test MCC, we developed an acute endogenous clearance (AEC) measurement. Leukocyte numbers in lung lavage fluid were enumerated in P-2119 and vehicle control groups. AEC was determined by quantifying an acute decrease in leukocyte numbers in lavage fluid. In addition, to quantify mucus elimination, distal airspace Muc5b was examined immunohistochemically by fixing air-inflated lungs via immersion in methacarn ${ }^{21,52}$. Fractions of randomly selected ventral alveolar airspaces occupied by Muc5b were assessed by point counting ${ }^{21}$.

Cytokine and chemokine assessment. Chemokines and cytokines were assessed using a 19-plex MSD Multi-spot Assay System in bronchoalveolar lavage fluid supernatant obtained from mice exposed to saline (untreated) or P-2119 treatment (treated). Data are presented in $\mathrm{pg} / \mathrm{ml}$ of each analyte (Supplementary Table 1). Statistical analysis was performed using a Mann-Whitney test.

Redox balance testing. Levels of glutathione (GSH) and oxidized glutathione (GSSG) were assess using a GSH-Glo ${ }^{\text {tw }}$ Glutathione Assay (Promega, Madison, WI). The protocol (384-well format) was modified from the manufacturer's protocol (96-well format, Promega V6911). In brief, $2.5 \mu$ of unclarified lung lavage samples (in duplicate) were treated with $2.5 \mu \mathrm{l} \mathrm{GSH}$-Glo reagent with or without TCEP (final concentration $=1 \mathrm{mM}$ ). Following a $30 \mathrm{~min}$ incubation at room temperature, luciferin detection reagent $(5 \mu \mathrm{l})$ was added, and luminescence signals were recorded on a plate reader (PerkinElmer EnVision 2104, $700 \mathrm{~nm}, 0.5 \mathrm{~s}$ exposure) 15 min later. For reactive oxygen species (ROS), samples were tested using a ROSGlo Assay (Promega). Lung lavage samples were prepared as described above for GSH-Glo assays. Following a 60 min incubation at room temperature, luciferin detection reagent $(5 \mu \mathrm{l})$ was added, and luminescence signals were recorded on a plate reader (PerkinElmer EnVision 2104, $700 \mathrm{~nm}, 0.5 \mathrm{~s}$ exposure) $20 \mathrm{~min}$ later.

Statistical analysis. Hydroxyproline, redox, inflammation, and gene expression data were analyzed by $t$-test or non-parametric analyses for non-normally distributed data, and parametric or non-parametric ANOVA's for multiple comparisons (GraphPad, La Jolla, CA). SHG percentage area histograms were fitted with Gaussian model using a least $\chi^{2}$ fit model to determine numbers of peaks in each experimental group in IgorPro (WaveMetrics, Lake Oswego, OR) and each pair was analyzed using a $t$-test. Degrees of freedom were over 100 for each distribution, giving a rejection region of 3.09 to $\infty$ for critical values (t) at a $0.1 \%$ level of significance.

\section{Data availability}

The data that support the findings of this study are available from the authors on reasonable request, see author contributions for specific data sets.
Received: 5 March 2018 Accepted: 14 November 2018

Published online: 18 December 2018

\section{References}

1. Raghu, G. et al. Diagnosis of idiopathic pulmonary fibrosis. An official ATS/ ERS/JRS/ALAT Clinical Practice Guideline. Am. J. Respir. Crit. Care. Med. 198, e44-e68 (2018)

2. King, T. E. Jr. et al. A phase 3 trial of pirfenidone in patients with idiopathic pulmonary fibrosis. N. Engl. J. Med. 370, 2083-2092 (2014).

3. Richeldi, L. et al. Design of the INPULSIS trials: two phase 3 trials of nintedanib in patients with idiopathic pulmonary fibrosis. Respir. Med. 108, 1023-1030 (2014)

4. Seibold, M. A. et al. A common MUC5B promoter polymorphism and pulmonary fibrosis. N. Engl. J. Med. 364, 1503-1512 (2011).

5. Evans, C. M. et al. Idiopathic pulmonary fibrosis: a genetic disease that involves mucociliary dysfunction of the peripheral airways. Physiol. Rev. 96, 1567-1591 (2016).

6. Rose, M. C. \& Voynow, J. A. Respiratory tract mucin genes and mucin glycoproteins in health and disease. Physiol. Rev. 86, 245-278 (2006).

7. Seibold, M. A. et al. The idiopathic pulmonary fibrosis honeycomb cyst contains a mucocilary pseudostratified epithelium. PLoS ONE 8, e58658 (2013)

8. Nakano, Y. et al. MUC5B promoter variant rs35705950 affects MUC5B expression in the distal airways in idiopathic pulmonary fibrosis. Am. J. Respir. Crit. Care. Med. 193, 464-466 (2016).

9. Helling, B. A. et al. Regulation of MUC5B expression in idiopathic pulmonary fibrosis. Am. J. Respir. Cell Mol. Biol. 57, 91-99 (2017).

10. Roy, M. G. et al. Muc5b is required for airway defence. Nature 505, 412-416 (2014).

11. Degryse, A. L. et al. Repetitive intratracheal bleomycin models several features of idiopathic pulmonary fibrosis. Am. J. Physiol. Lung Cell Mol. Physiol. 299, L442-L452 (2010).

12. Button, B. et al. A periciliary brush promotes the lung health by separating the mucus layer from airway epithelia. Science 337, 937-941 (2012).

13. Sloane, P. A. et al. A pharmacologic approach to acquired cystic fibrosis transmembrane conductance regulator dysfunction in smoking related lung disease. PLoS ONE 7, e39809 (2012)

14. Chu, K. K. et al. In vivo imaging of airway cilia and mucus clearance with micro-optical coherence tomography. Biomed. Opt. Express 7, 2494-2505 (2016).

15. Solomon, G. M. et al. Assessment of ciliary phenotype in primary ciliary dyskinesia by micro-optical coherence tomography. JCI Insight 2, e91702 (2017).

16. Birket, S. E. et al. A functional anatomic defect of the cystic fibrosis airway. Am. J. Respir. Crit. Care. Med. 190, 421-432 (2014).

17. Johnson M. R., Thelin W. R. Novel monothiol mucolytic agents. Patent WO2016123335A1 (2016)

18. Moeller, A., Ask, K., Warburton, D., Gauldie, J. \& Kolb, M. The bleomycin animal model: a useful tool to investigate treatment options for idiopathic pulmonary fibrosis? Int. J. Biochem. Cell Biol. 40, 362-382 (2008)

19. Fahy, J. V. \& Dickey, B. F. Airway mucus function and dysfunction. N. Engl. J. Med. 363, 2233-2247 (2010).

20. Button, B., Anderson, W. H. \& Boucher, R. C. Mucus hyperconcentration as a unifying aspect of the chronic bronchitic phenotype. Ann. Am. Thorac. Soc. 13, S156-S162 (2016)

21. Evans, C. M. et al. The polymeric mucin Muc5ac is required for allergic airway hyperreactivity. Nat. Commun. 6, 6281 (2015).

22. Peljto, A. L. et al. Association between the MUC5B promoter polymorphism and survival in patients with idiopathic pulmonary fibrosis. JAMA 309, 2232-2239 (2013)

23. Molyneaux, P. L. et al. The role of bacteria in the pathogenesis and progression of idiopathic pulmonary fibrosis. Am. J. Respir. Crit. Care Med. 190, 906-913 (2014).

24. Fernandez-Blanco, J. A., et al. Attached stratified mucus separates bacteria from the epithelial cells in COPD lungs. JCI Iinsight 3, 906-913 (2018).

25. Bauer, A. K., et al. Requirement for MUC5AC in KRAS-dependent lung carcinogenesis. JCI Insight 3, 120941 (2018).

26. Livraghi-Butrico, A. et al. Contribution of mucus concentration and secreted mucins Muc5ac and Muc5b to the pathogenesis of muco-obstructive lung disease. Mucosal Immunol. 10, 829 (2017)

27. Evans, C. M. et al. Mucin is produced by clara cells in the proximal airways of antigen-challenged mice. Am. J. Respir. Cell Mol. Biol. 31, 382-394 (2004).

28. Zhu, Y. et al. Munc13-2-/- baseline secretion defect reveals source of oligomeric mucins in mouse airways. J. Physiol. 586, 1977-1992 (2008). 
29. Reader, J. R. et al. Pathogenesis of mucous cell metaplasia in a murine asthma model. Am. J. Pathol. 162, 2069-2078 (2003).

30. Raju, S. V. et al. The cystic fibrosis transmembrane conductance regulator potentiator ivacaftor augments mucociliary clearance abrogating cystic fibrosis transmembrane conductance regulator inhibition by cigarette smoke. Am. J. Respir. Cell Mol. Biol. 56, 99-108 (2017).

31. Boucher, R. C. Idiopathic pulmonary fibrosis-a sticky business. N. Engl. J. Med. 364, 1560-1561 (2011).

32. Williamson, J. D., Sadofsky, L. R. \& Hart, S. P. The pathogenesis of bleomycininduced lung injury in animals and its applicability to human idiopathic pulmonary fibrosis. Exp. Lung Res. 41, 57-73 (2015).

33. Peng, R. et al. Bleomycin induces molecular changes directly relevant to idiopathic pulmonary fibrosis: a model for "active" disease. PLOS ONE 8, e59348 (2013).

34. Yang, I. V. et al. Expression of cilium-associated genes defines novel molecular subtypes of idiopathic pulmonary fibrosis. Thorax 68, 1114-1121 (2013).

35. Pardo, A. et al. Up-regulation and profibrotic role of osteopontin in human idiopathic pulmonary fibrosis. PLoS Med. 2, e251 (2005).

36. Gharib, S. A. et al. Matrix metalloproteinase-7 coordinates airway epithelial injury response and differentiation of ciliated cells. Am. J. Respir. Cell Mol. Biol. 48, 390-396 (2013).

37. Anderson, W. H. et al. The relationship of mucus concentration (Hydration) to mucus osmotic pressure and transport in chronic bronchitis. Am. J. Respir. Crit. Care. Med. 192, 182-190 (2015).

38. Hunninghake, G. M. et al. MUC5B promoter polymorphism and interstitial lung abnormalities. N. Engl. J. Med. 368, 2192-2200 (2013).

39. Araki, T. et al. Development and progression of interstitial lung abnormalities in the Framingham Heart Study. Am. J. Respir. Crit. Care Med. 194, 1514-1522 (2016).

40. Kropski, J. A. et al. Extensive phenotyping of individuals at risk for familial interstitial pneumonia reveals clues to the pathogenesis of interstitial lung disease. Am. J. Respir. Crit. Care Med. 191, 417-426 (2015).

41. Voltz, J. W. et al. Male sex hormones exacerbate lung function impairment after bleomycin-induced pulmonary fibrosis. Am. J. Respir. Cell Mol. Biol. 39, 45-52 (2008).

42. Redente, E. F. et al. Age and sex dimorphisms contribute to the severity of bleomycin-induced lung injury and fibrosis. Am. J. Physiol. Lung Cell Mol. Physiol. 301, L510-L518 (2011).

43. Ochs, M. \& Muhlfeld, C. Quantitative microscopy of the lung: a problembased approach. Part 1: basic principles of lung stereology. Am. J. Physiol. Lung Cell Mol. Physiol. 305, L15-L22 (2013).

44. Liu, L. et al. Method for quantitative study of airway functional microanatomy using micro-optical coherence tomography. PLoS ONE 8, e54473 (2013).

45. Han, J. C. \& Han, G. Y. A procedure for quantitative determination of tris(2carboxyethyl)phosphine, an odorless reducing agent more stable and effective than dithiothreitol. Anal. Biochem. 220, 5-10 (1994).

46. Kirkham, S., Sheehan, J. K., Knight, D., Richardson, P. S. \& Thornton, D. J. Heterogeneity of airways mucus: variations in the amounts and glycoforms of the major oligomeric mucins MUC5AC and MUC5B. Biochem. J. 361, 537-546 (2002).

47. Thornton, D. J., Carlstedt, I. \& Sheehan, J. K. Identification of glycoproteins on nitrocellulose membranes and gels. Methods Mol. Biol. 32, 119-128 (1994).

48. Duncan, G. A. et al. Microstructural alterations of sputum in cystic fibrosis lung disease. JCI Insight 1, e88198 (2016).

49. Hill, D. B. et al. A biophysical basis for mucus solids concentration as a candidate biomarker for airways disease. PLoS ONE 9, e87681 (2014).

50. Seagrave, J., Albrecht, H. H., Hill, D. B., Rogers, D. F. \& Solomon, G. Effects of guaifenesin, $\mathrm{N}$-acetylcysteine, and ambroxol on MUC5AC and mucociliary transport in primary differentiated human tracheal-bronchial cells. Respir. Res. 13, 98 (2012).

51. Matsui, H. et al. A physical linkage between cystic fibrosis airway surface dehydration and Pseudomonas aeruginosa biofilms. Proc. Natl Acad. Sci. USA 103, 18131-18136 (2006).
52. Johansson, M. E. et al. The inner of the two Muc2 mucin-dependent mucus layers in colon is devoid of bacteria. Proc. Natl Acad. Sci. USA 105, 15064-15069 (2008).

\section{Acknowledgements}

The authors thank Dr. Jennifer Matsuda at National Jewish Health for generating Muc5b transgenic mice, Melanie Sawyer at Parion Sciences for assistance with in vitro mucolytic testing, and Jake McDonald at Lovelace Respiratory Research Institute for support with aerosol delivery. This research was supported by the National Heart, Lung and Blood Institute (UH2/3-HL123442, R01-HL097163, R01-HL080396, R01-HL130938, R21/R33HL120770, R35-HL135816, and P01-HL092870, P01-HL108808), National Institute of Diabetes and Digestive and Kidney Diseases (P30-DK072482, P30DK065988), Department of Defense (W81XWH-17-0597), Cystic Fibrosis Foundation HILL16XXO and BOUCHER15RO, and Parion Sciences, Inc.

\section{Author contributions}

L.A.H., G.M.S., W.R.T., I.V.Y., C.M.E., and D.A.S. designed the study; L.A.H., C.E.H., E.D., A.E., N.H., and M.I.S. designed, performed, and analyzed experiments. G.M.S., G.J.T., and S.M.R. designed, performed, and analyzed $\mu$ OCT experiments. D.B.H., W.J. K., and M.R.M. designed, performed, and analyzed mucus biophysical and mucin molecular weight assays. D.E.G.V. M.E.V. and W.R.T. synthesized and analyzed P2119. K.S.C. and Y.S. performed analyses of redox balance. L.A.H. and D.A.S. wrote the manuscript with help from all coauthors. C.E.H. and D.A.S. supervised the manuscript preparation.

\section{Additional information}

Supplementary Information accompanies this paper at https://doi.org/10.1038/s41467018-07768-9.

Competing interests: D.A.S. is the founder and chief scientific officer of Eleven P15, a company focused on the early diagnosis and treatment of pulmonary fibrosis. D.A.S. has patents awarded (US Patent no: 8,673,565) and submitted (US Patent application no: 62/ 250,390, US Patent application no: 62/525,087, and US Patent application no: 62/ $525,088)$ for the treatment and diagnosis of fibrotic lung disease. D.E.G.V., M.E.V. and W.R.T. are employees of Parion Sciences. The remaining authors declare no competing interests.

Reprints and permission information is available online at http://npg.nature.com/ reprintsandpermissions/

Publisher's note: Springer Nature remains neutral with regard to jurisdictional claims in published maps and institutional affiliations.

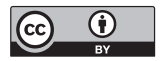

Open Access This article is licensed under a Creative Commons Attribution 4.0 International License, which permits use, sharing, adaptation, distribution and reproduction in any medium or format, as long as you give appropriate credit to the original author(s) and the source, provide a link to the Creative Commons license, and indicate if changes were made. The images or other third party material in this article are included in the article's Creative Commons license, unless indicated otherwise in a credit line to the material. If material is not included in the article's Creative Commons license and your intended use is not permitted by statutory regulation or exceeds the permitted use, you will need to obtain permission directly from the copyright holder. To view a copy of this license, visit http://creativecommons.org/ licenses/by/4.0/.

(C) The Author(s) 2018 